\title{
Applying the Evidence in Treatment of Acute Uncomplicated Bronchitis in Emergency Medicine
}

\author{
Langer K*, Steele D, Schnepper L and Fritzlar S \\ Winona State University, USA
}

*Corresponding author: Kimberly Langer, Winona State University, 859 30th Ave SE

Rochester, MN 55904, USA, Tel: 715-523-9566; Email: klanger@winona.edu

\section{Research Article \\ Volume 3 Issue 6}

Received Date: October 18, 2019

Published Date: November 07, 2019

DOI: $10.23880 /$ nhij-16000207

\section{Abstract}

Studies demonstrate that $95 \%$ of acute uncomplicated bronchitis cases are viral in nature. Emergency medicine literature indicates that between $42.2 \%$ and $75 \%$ of patients diagnosed with acute, uncomplicated bronchitis are prescribed antibiotics. This is a healthcare concern, as the inappropriate use of antibiotic therapy is a major driver causing antibiotic resistance. Additionally, evidence-based guidelines are not supportive of radiological studies as a diagnostic modality for acute uncomplicated bronchitis.

A review of literature including clinical care guidelines recommend the use of supportive care for the treatment of acute uncomplicated bronchitis. Specifically, the clinical care guidelines do not support antimicrobial therapy. Objectively, if normal vital signs are noted, with lack of adventitious breath sounds, clinical care guidelines do not support the use of radiological imaging.

A Quality Improvement project was implemented at a large, urban emergency care center in the Upper Midwest. Multifaceted evidence-based education regarding the diagnosis of acute uncomplicated bronchitis and management was provided to emergency medicine providers staffing a large urban (Level III trauma center) emergency department.

Data were collected from October 2014-Februray $2015(\mathrm{~N}=90)$ this data were compared to data collected during the study period, of October 2015-Februray 2016 (N=49). Pre-intervention antibiotic prescription rate was 49.4\%. Post intervention prescription rate was $8.2 \%, \mathrm{p}<0.0001$. A reduction in the number of radiological studies was noted to be from $90 \%$ to $67 \%, \mathrm{p}=0.0021$.

The results of this study suggest that multifaceted brief educational sessions can be effective in the promotion and implementation of evidence-based care. Additional educational efforts to promote evidence-based care in the management of other acute conditions maybe successful using this model, and should be explored.

Keywords: Emergency medicine; Evidence-base practice; Bronchitis 


\section{Nursing \& Healthcare International Journal}

Abbreviations: VRE: Vancomycin-Resistant Enterococcus; MRSA: Methicillin-Resistant Staph Aureus; CDC: Center for Disease Control; PCV-7: Pneumococcal Conjugate Vaccine; APPs: Advanced Practice Providers; ESI: Emergency Severity Index.

\section{Introduction}

\section{Problem Description}

Studies show that $95 \%$ of cases of acute bronchitis, in otherwise healthy adults, are determined to be viral in nature [1,2]. Prescribers continue to utilize antibiotic therapy for this particular diagnosis [2,3]. Specifically, in primary care offices, approximately $75 \%$ of all prescriptions written are for upper respiratory infections [1]. In emergency care settings, antibiotic therapy for upper respiratory infections ranged from $42 \%$ [3] to $75 \%$ [2]. $\mathrm{Xu}$, et al. [4] analyzed 2.2 million cases of uncomplicated upper respiratory infections in emergency department settings in the United States during the years 2007-2008. Results demonstrated a 52\% antibiotic prescription rate and $51 \%$ of the patients had a diagnosis of acute bronchitis. Of the antibiotics prescribed, 33\% received macrolides, and $50 \%$ of the patients had radiological studies performed, which was commonly a chest X-ray.

An ongoing healthcare concern is antibiotic resistance. "The use of antibiotics is the main driver in creating selective pressure for the emergence of antibiotic resistant bacteria" [1]. Lee, et al. [1] estimated that over $50 \%$ of antibiotic prescriptions written were not indicated for the prescribed diagnosis. Antibiotic resistant diseases have been and continue to be identified. These include: vancomycin-resistant enterococcus (VRE), methicillin-resistant staph aureus (MRSA), and metronidazole-resistant Clostridium difficile, all of which resulted from overuse of antibiotics [5]. With ongoing resistance to bacterial microorganisms, researchers are now noticing an increase in resistant respiratory pathogens. According to Jenkins, et al. [6], pneumococcal pneumonia resistance to macrolides had held stable at $30 \%$ but now has trended upward to $36 \%$ over a three year span (2003-2006) in the United States $(\mathrm{p}<.0001)$. According to the Center for Disease Control (CDC), resistance of $>30 \%$ is of concern as this poses a serious threat to the treatment of specific diagnoses [5]. Specifically noted by the CDC, pneumococcal pneumonia resistance is a serious threat to the treatment of pneumonia [5].
However, the CDC estimates that each year "in the United States, at least two million people acquire serious infections with microorganisms that are resistant to one or more of the antibiotics designed to treat those specific infections and at least 23,000 people die from those antibiotic resistant infections" [5]. The total economic cost of antibiotic resistance in the U.S. has been calculated at a high of $\$ 20$ billion of direct healthcare costs, which was noted in 2008. Additional costs for lost productivity were in excess of $\$ 35$ billion a year [5].

Initiatives have been implemented in the United States to reduce the overuse of antibiotics. The CDC started one of the first major initiatives "Get Smart: Know when Antibiotics Work" in 1995 [7]. After initiation of the "Get Smart: To know when Antibiotics work" campaign, antibiotic prescriptions written for pediatric population did decline by $18 \%$ [1]. This is data supported the use of the educational campaigns of the 1990's as well as other interventions including supportive care and patient education. The interventions included: introduction of routine immunizations for infants and children which included the pneumococcal conjugate vaccine (PCV-7). However, in the adult population, the prescription rate remained the same, and in the older adult the prescription rate actually increased by $30 \%$ [1]. With the continued emergence of new antibiotic resistant diseases and pneumococcal pneumonia becoming more drug-resistant, more initiatives are needed towards improvement of antibiotic stewardship.

Furthermore, radiological studies have been used as a means of diagnosis of upper respiratory infections including bronchitis and pneumonia, etc. These studies have risks and benefits, as with antibiotic therapy. Carcinogenesis and radiation exposure remain the most common risks. Diagnostic X-rays remain the largest manmade source of radiation exposure to the general population [8]. A posterior-anterior and lateral chest Xray delivers an average of 0.06 to $0.25 \mathrm{mSv}$ per study [9]. The average amount of background radiation in the United States in 2006 was $3.0 \mathrm{mSv}$ [10]. Despite radiological studies with potential exposure to higher doses of radiation (i.e.: Cat Scan), the risk is minimal and the risk/benefit ratio favors the patient. As with the issue with antibiotic therapy, the problem occurs when radiological studies are overused or inappropriately ordered without evidence of clinical need [11]. Based on this information, the opportunity presented to use this data to develop and implement a quality improvement project at a large, urban emergency care centre in the Upper Midwest. Multifaceted evidence-based education 


\section{Nursing \& Healthcare International Journal}

regarding the diagnosis of acute uncomplicated bronchitis and management was provided to emergency medicine providers with the goal to reduce the number of antibiotic prescriptions written and radiological studies ordered.

\section{Available Knowledge}

\section{Literature Search Strategy}

A literature search was performed to help initiate the quality improvement project. Specific search engines that were utilized included: Google Scholar, PubMed, and CINAHL. Search terms included: Acute Bronchitis, Treatment, Antibiotic Use, and Clinical Guidelines for the treatment of Acute Bronchitis, Supportive Care, Upper Respiratory Infection, Chest X-ray, and Radiological Studies. There were over 220,000 articles that were found. Of the 220,000 articles, 90 articles were reviewed and ultimately, 14 articles were used for this Quality Improvement project. Data outcomes, use of broadspectrum antibiotics, adult population, and articles published from 1995-2015 were the primary inclusion criteria for this literature review. Although there is a 20year span in the search of articles, many of the articles that were found built off of prior articles and clinical guidelines. A limiting factor of this literature review was that many of the reviewed articles focused on information/data of a specific treatment modality for acute bronchitis with the level of evidence associated with each of the treatment modalities. During the initial literature search, the focus was on identification of the current standard of care for the treatment of acute uncomplicated bronchitis. Studies reviewed included randomized controlled trials, cohort studies, clinical guidelines, and meta-analysis. A second literature search done using the same keywords and strategy as mentioned prior but with an aim to review clinical care guidelines related to the treatments to acute uncomplicated bronchitis.

After implementation of the project, a subsequent literature search was performed to enhance and add to the current studies that were reviewed. Similar search criteria and search engines were used as noted above. Within this search, studies outside of the United States were reviewed, noting that a similar problem exists elsewhere. A downfall to this review was that many of the studies were the same or similar when searched, but listed under different headings or found using different search terms. This slightly limited our search but still allowed a variety of evidence and articles.
A third search method used was review of up to date research articles that were received by both authors via email updates. Both authors are practicing providers and have subscriptions to various medical journals and professional practice websites.

Within the literature search, as noted above, clinical guidelines for treatment of acute bronchitis were reviewed based on applicability to the patient population for uncomplicated bronchitis. Each guideline was reviewed using the AGREE II tool which helps determine the quality of the clinical guideline. The AGREE II tool also provides direction to aid with the development of new clinical guidelines as well as how information should be reported in clinical guidelines [12].

\section{Literature Review}

Literature review included four randomized controlled trials of which three had published results. The fourth study was a template study that was never put into practice, thus no data were available. Of the three randomized controlled trials, all had similar interventions and outcomes. The interventions included placing subjects into study arms for which antibiotic therapy was provided versus no antibiotic therapy. Results showed similar outcomes in which there was no significant change in the patient outcome [13-15].

Gonzales, et al. [16] conducted a study, in which providers and patients were followed for a period of time. From a prescriber perspective, their prescription habits were reviewed for their treatment of acute bronchitis. During the second year of this study, patients and prescribers were provided with educational materials about antibiotic therapy and appropriate uses. After this specific intervention, there was a decrease in the number of prescriptions written for antibiotics for acute bronchitis at the study site versus the control site. A prospective research template created by Altiner, et al. [17] that had not been implemented was rated as probably effective as the framework was similar to the already reviewed studies. A survey review by Stone, et al. [3] was also listed as possibly effective as this was a review of data, which viewed a specific intervention, not necessarily looking into a new intervention.

One final review was of the Cochrane Review, which was done in regards to "Antibiotics for Acute Bronchitis." From this review, seventeen trials were included in the analysis. Based on the results, there were limited data to support the use of antibiotics for the treatment of acute 


\section{Nursing \& Healthcare International Journal}

bronchitis. These studies also had follow-up data with the study participants that yielded no change in their symptomatology nor was there any change in their clinical status between the antibiotic and placebo groups [14].

The literature regarding radiological studies and acute uncomplicated bronchitis was sparse. A study by Cao, et al. [18] showed two randomized control trials involving 2024 patients. However, 522 of these patients were children. Based on this study, it was resulted that chest radiographs did not result in a better clinical outcome for patients (duration of illness and of symptoms) with acute lower respiratory tract infections. Their definition of acute chest infections is as stated: "Acute chest infections (lower respiratory tract infections) such as pneumonia, bronchitis and bronchiolitis...." [18]. This particular study included bronchitis as a lower respiratory tract infection, even though it typically is classified as an upper respiratory infection.

Discrepancy could be noted within the articles chosen as this project was aimed to reduce antibiotic prescription rate for the diagnosis of acute bronchitis. As noted, studies that focused on the scope of practice of the provider writing the prescriptions, randomized trials to review different interventions including communication to providers and patients as well as educational material, and data reviewed support the proposed problem has been an on-going issue for some time.

\section{Review of Clinical Treatment Guidelines}

Six clinical guidelines were identified through the literature search and reviewed. After appraising the AGREE II tool results of each guideline reviewed, the authors determined that there was a wide variation among the guidelines in terms of strength, overall development, and the ability to easily place the guidelines into clinical practice.

\section{Rationale}

The theory used to help facilitate this quality improvement project was directed by Roger's Diffusion of Innovations theory. "An innovation is an idea, practice, or project that is perceived as new by an individual or other unit of adoption" [19]. Rogers described the innovationdecision process as "an information-seeking and information-processing activity, where an individual is motivated to reduce uncertainty about the advantages and disadvantages of an innovation" (p. 172). The innovation-decision process involves five steps: (1) knowledge, (2) persuasion, (3) decision, (4) implementation, and (5) confirmation. These stages typically follow each other in a time-ordered manner [19].

\section{Specific Aims}

The literature review demonstrated that bronchitis is a viral process in $95 \%$ of patients $[1,2]$. Antibiotics should be considered in high-risk categories, which include; age $>65$ years, restricted pulmonary disease, or immunocompromised patient [20]. Evidence-based guidelines recommend antibiotic therapy not be prescribed for the diagnosis of acute uncomplicated bronchitis [14,21-24]. Radiological studies are not clinically indicated if the patient is clinically stable, afebrile, and has no signs of adventitious breath sounds [25].

The clinical question after the literature review was: In providers managing acute uncomplicated bronchitis in an emergency care centre, would a multifaceted, evidence based education intervention improve evidence based practice, measured by comparison of antibiotic prescriptions written and radiologic studies ordered, pre and post intervention.

Population: Providers (Nurse Practitioners, Physician Assistants and Physicians) managing patients with acute uncomplicated bronchitis in an emergency department.

Intervention: Multifaceted evidence-based education on acute uncomplicated bronchitis diagnosis and management.

Comparison: The percentage of patients diagnosed with acute uncomplicated bronchitis, prescribed antibiotic therapy and/or order radiological studies before the educational intervention compared to the percentage of patients diagnosed with acute uncomplicated bronchitis, prescribed antibiotic therapy and/or order radiological studies after implementation of the interventions.

Outcome: The change in the percentage of antibiotics prescribed and radiological studies ordered for acute uncomplicated bronchitis after an evidence-based education intervention.

\section{Purpose of the project}

The introduction of this paper has demonstrated some major themes. Bronchitis is largely a viral process [1]. In primary care, antibiotic prescriptions are written for upper respiratory diagnoses (which include bronchitis) $75 \%$ of the time [1], and in emergency medicine literature, antibiotics are written for bronchitis between 42.2\% [3] and $75 \%$ [2]. Antibiotic resistance develops when antibiotics are not used appropriately [1]. Lee, et al. [1] 


\section{Nursing \& Healthcare International Journal}

found that $50 \%$ of prescriptions written for antibiotics were for the wrong indication. Radiological studies are not indicated in diagnosing acute uncomplicated bronchitis when the patient is clinically stable, afebrile, with clear lung sounds [25]. The purpose of this project was to implement a quality improvement project to promote evidence-based practice for the diagnosis and management of acute uncomplicated bronchitis.

\section{Methods}

\section{Context}

The intervention of this study was implemented at a large, suburban emergency care center where there are 60,000 patient visits each year. Based on the location of the project site, there are wide arrays of patients seen. This includes Caucasian, African American, Asian American, American Indian, and others.

A meeting with key stakeholders prior to the project initiation allowed for collaboration with the emergency department staff, the Quality Improvement Director, as well as other medical staff. After this meeting, all resources needed were identified and granted. These resources included: meeting with the emergency department staff, retrospective chart review (prior to intervention), and access to the emergency department. Leadership were very forthcoming and supportive of the project.

\section{Interventions}

The educational intervention was implemented in a busy urban emergency department in the upper Midwest. The intervention was a lecture format on acute uncomplicated bronchitis provided to the health care providers, consisting of Physician Assistants, Nurse Practitioners and Physicians. This occurred during a mandatory provider meeting. A voice over lecture was also being posted to the educational intranet of the sponsoring organization. The intervention included; pathophysiology of bronchitis, literature review, review of evidence-based guidelines, and appropriate treatment. A discussion of antibiotic stewardship was also included. Additionally, ongoing bi-weekly email blasts were also implemented during the study phase to remind providers, and re-educate, regarding the evidence behind bronchitis, treatment guidelines, and facts about antibiotic resistance. Within the "fast track" rooms of the department, posters from the Center from Disease Control and Prevention were placed (Figures $1 \& 2$ ). These reminded providers, and educated patients, on the viral nature of

Langer $\mathrm{K}$, et al. Applying the Evidence in Treatment of Acute Uncomplicated Bronchitis in Emergency Medicine. Nurs Health Care Int J 2019, 3(6): 000207. colds/flu/bronchitis. Of note, many of the cases of bronchitis have a low acuity thus not warranting a general emergency room type setting but more of a "fast track" or urgent care type setting. Lastly an algorithm was devised and implemented based of the six treatment guidelines that were reviewed and placed in providers' work stations (Figure 3). The intervention study period was between October 1, 2015 and February 28, 2016.

Benefits of this multifaceted intervention include: multi-cultural, inexpensive, time manageable and effective strategies based on review of the evidence. Risks to successful implementation were potential low turnout of providers at the mandatory meeting where initial education was provided. This was mitigated by email reminders biweekly, and posting the intervention in a voice over PowerPoint on medical provider's educational website.

\section{Study of the Intervention}

A pre-intervention chart review was completed. Charts were reviewed from October 1, 2014 through March 1, 2015. On September 15, 2015, an educational session was held to provide supporting literature review and data of our project. On October 1, 2015, the treatment algorithm was displayed in the work area of the prescribers. Educational posters were placed in the patient exam rooms. Subsequently, the study of the intervention was a retrospective chart review from October 1, 2015 until February 28, 2016. The data was then compared using appropriate statistical analysis to determine if there was a statistical significant change between pre and post intervention groups. The goal was to reduce rates of both prescriptions written and radiological studies ordered by $20 \%$. This figure was estimated through the literature review as a realistic goal as most studies seemed to achieve this level of reduction $[13,26,27]$.

In reviewing the literature regarding the best evidence for management of acute uncomplicated bronchitis included no radiological studies and no antibiotic prescriptions for diagnosis and treatment, respectively. The literature supported a multi-faceted approach with multiple interventions versus a single intervention. This evidence-based project had a total of four primary interventions.

- Educational program (both live and online) regarding acute uncomplicated bronchitis and up to date evidence regarding this diagnosis. The online portion was available even though the face to face education was mandatory for all providers to attend. Online, with 
an email reminder, afforded most providers to get the educational session.

- Bimonthly email reminders were also initiated during the study period to remind providers of the viral nature of the acute bronchitis, evidence-based guidelines, antibiotic stewardship, and reminders of the education online session. This intervention was chosen to keep project in the forefront of providers practice.

- Posters were placed in the rooms where acute uncomplicated bronchitis patients typically treated. (Figures $1 \& 2$ ). These posters were obtained from the CDC website. This type of intervention was chosen to aid in educating patients as well as to serve as an educational tool for providers when explaining the diagnosis.

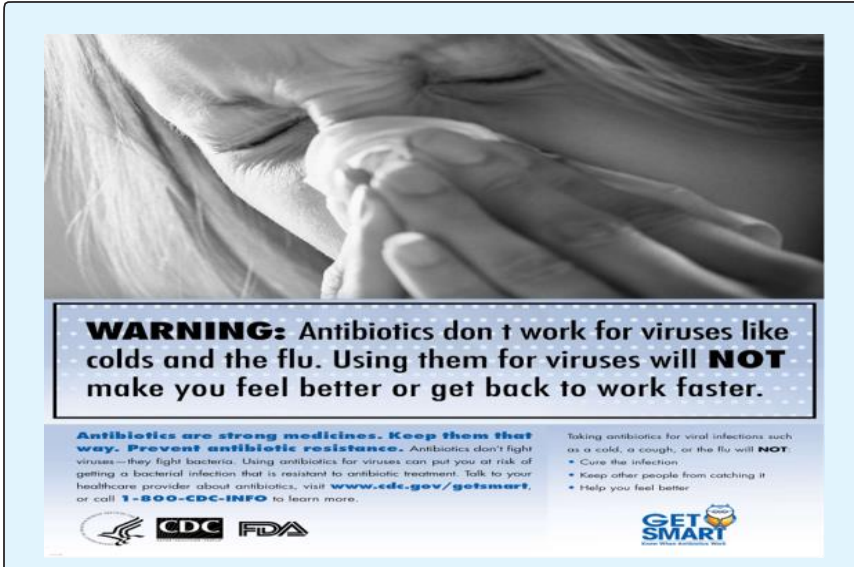

Figure 1: Poster placed in exam room during study [28].

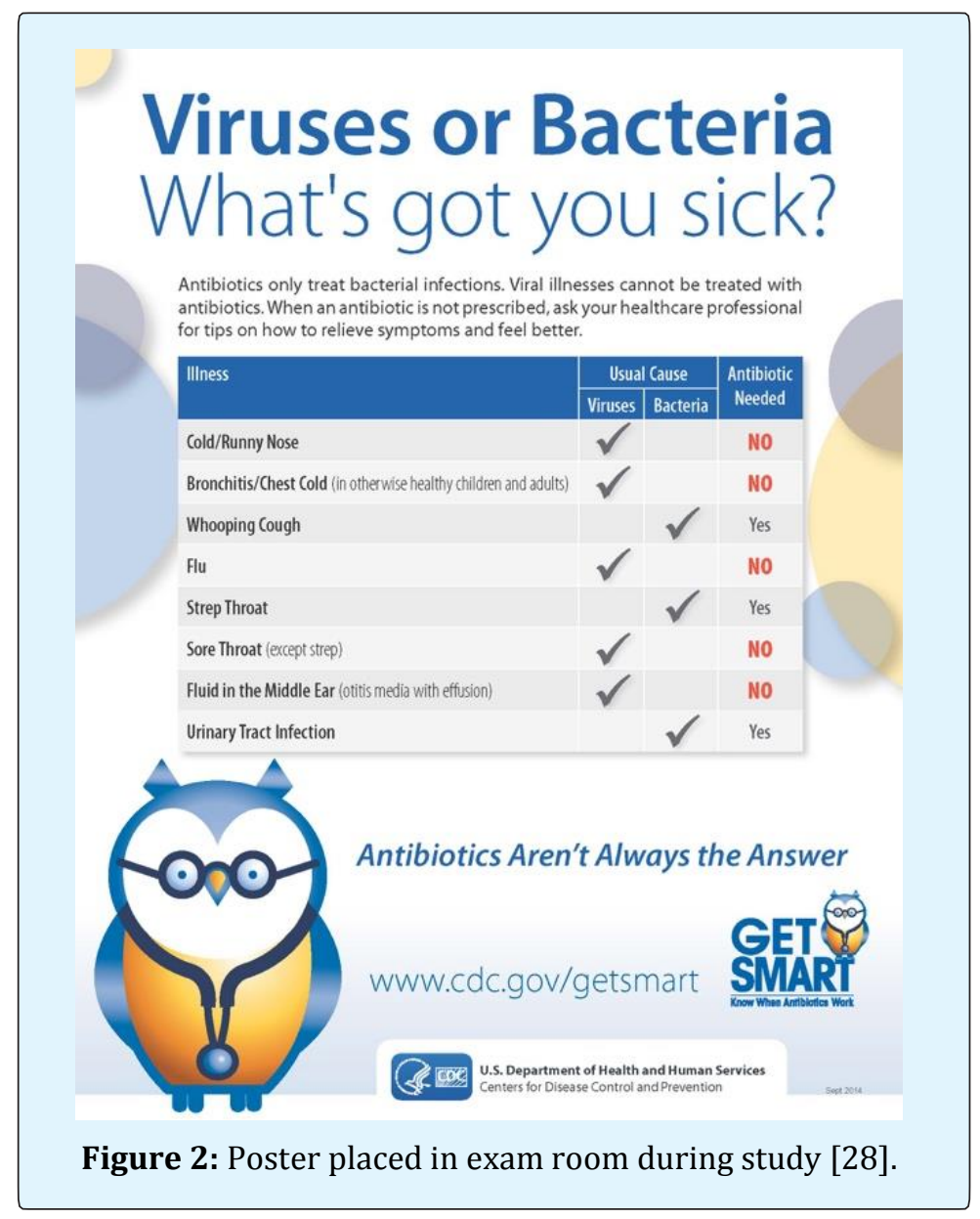

- An algorithm was designed by the authors, based on the six treatment guidelines that were reviewed and then it was posted in the providers' work stations (Figure 3). 


\section{Nursing \& Healthcare International Journal}

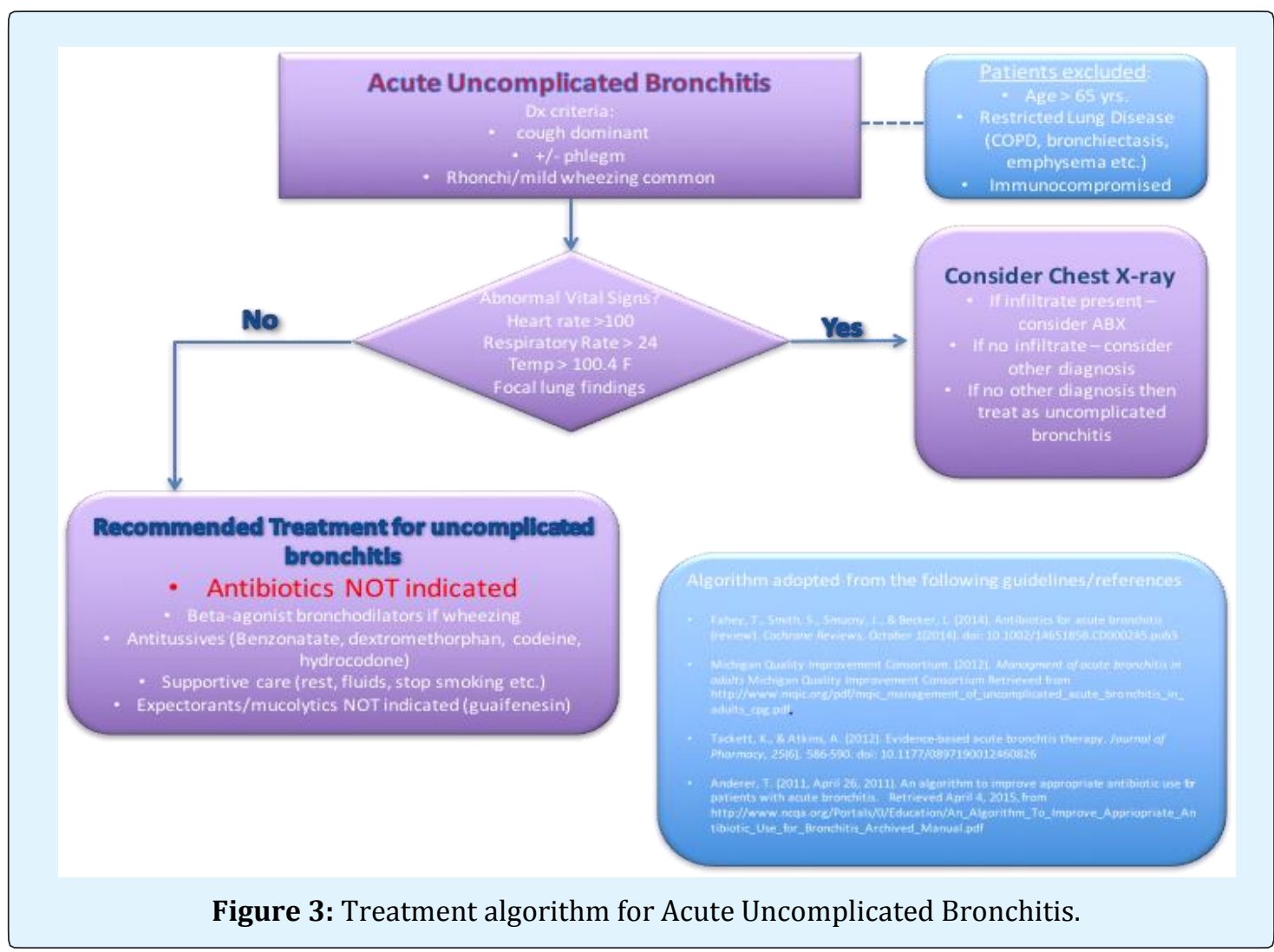

\section{Measures}

The measure of this project looked at applying the evidence to care for patients with acute uncomplicated bronchitis in an emergency department setting. The definition that was used for acute uncomplicated bronchitis which drove the study inclusion and exclusion criteria was based on the literature review. This included patients between the ages of 16 and 64 years of age. Exclusion criteria included; age younger than 16 or older than 65 , restrictive lung disease, and immunosuppressed patients. A pre-intervention retrospective chart review from October 1, 2014 through March 1, 2015, found that $49 \%$ of patients were given antibiotics for the diagnosis of acute bronchitis. Additionally, $90 \%$ of patients had radiological studies ordered.

\section{Data Collection Process and Logistics}

Initial data collection took place through a retrospective chart review from October 1, 2014 through March 1, 2015; this provided the pre-intervention data in terms of antibiotic prescriptions written and radiological studies ordered for acute uncomplicated bronchitis. Post- intervention data were gathered from October 1, 2015 February 28, 2016. The data were obtained from EPIC (electronic medical record) encrypted to ensure security.

\section{Limiters Included}

The diagnosis of bronchitis and bronchitis not specified, using ICD 9 codes (pre-intervention). The health care system switched to ICD 10 codes during the project period. During the post study phase ICD-10 codes were used. The diagnosis codes of acute bronchitis (J20) and bronchitis not specified (J40).

Data were reviewed and erroneous records were removed from the data set. Co-morbid conditions were reviewed and categories of "complicated bronchitis" were removed including, age less than 16 or greater than 65 , restrictive lung disease (chronic obstructive pulmonary disease, emphysema), and immunosuppressed patients.

\section{Analysis}

Statistical Analysis included: Fischer's exact test, confidence intervals for the difference in proportions and 
relative risk, of antibiotics and radiological studies pre and post intervention. JMP statistical software was used to analyze the data and perform the stated statistical tests [29]. Confidence intervals were also calculated for the difference in proportions. For this project the $95 \%$ confidence interval was used [30].

\section{Ethical Considerations}

Ethical considerations included the following: patient confidentiality, data collection which included provider and patient's information, access to patients medical records were all aspects of consideration during this project.

\section{Results}

\section{Pre-Intervention}

An initial survey, of the emergency department providers, was conducted to assess their understanding of the evidence supporting the appropriate treatment of acute uncomplicated bronchitis. This survey was used to assess the providers awareness of clinical care guidelines and evidence-based care aimed at treatment of acute uncomplicated bronchitis. The overall completion rate of the survey was $67 \%$. Forty percent of respondents were physicians and $60 \%$ were advanced practice providers (APPs) (Nurse Practitioners/Physician Assistants). Based on the survey results, $93 \%$ identified they practiced based on evidence-based guidelines, $100 \%$ denied prescribing antibiotics for acute bronchitis, and $66 \%$ identified awareness of clinical practice guidelines for acute bronchitis.

The pre-intervention chart review demonstrated that $49.4 \%(n=90)$ of patients with the diagnosis of acute uncomplicated bronchitis did receive antibiotics. In addition, of the charts reviewed, $90 \%$ of the patients had radiological studies performed.

Results from a pre-intervention survey and chart review found that the perceived standard of care for acute bronchitis and what providers were actually doing were quite different. These data were brought forth at the educational meeting.

The evidence-based quality improvement project took place over one year, March 2015 to March 2016. Please refer to Figure 4 for the timeline of implementation of this evidence-based quality improvement project.

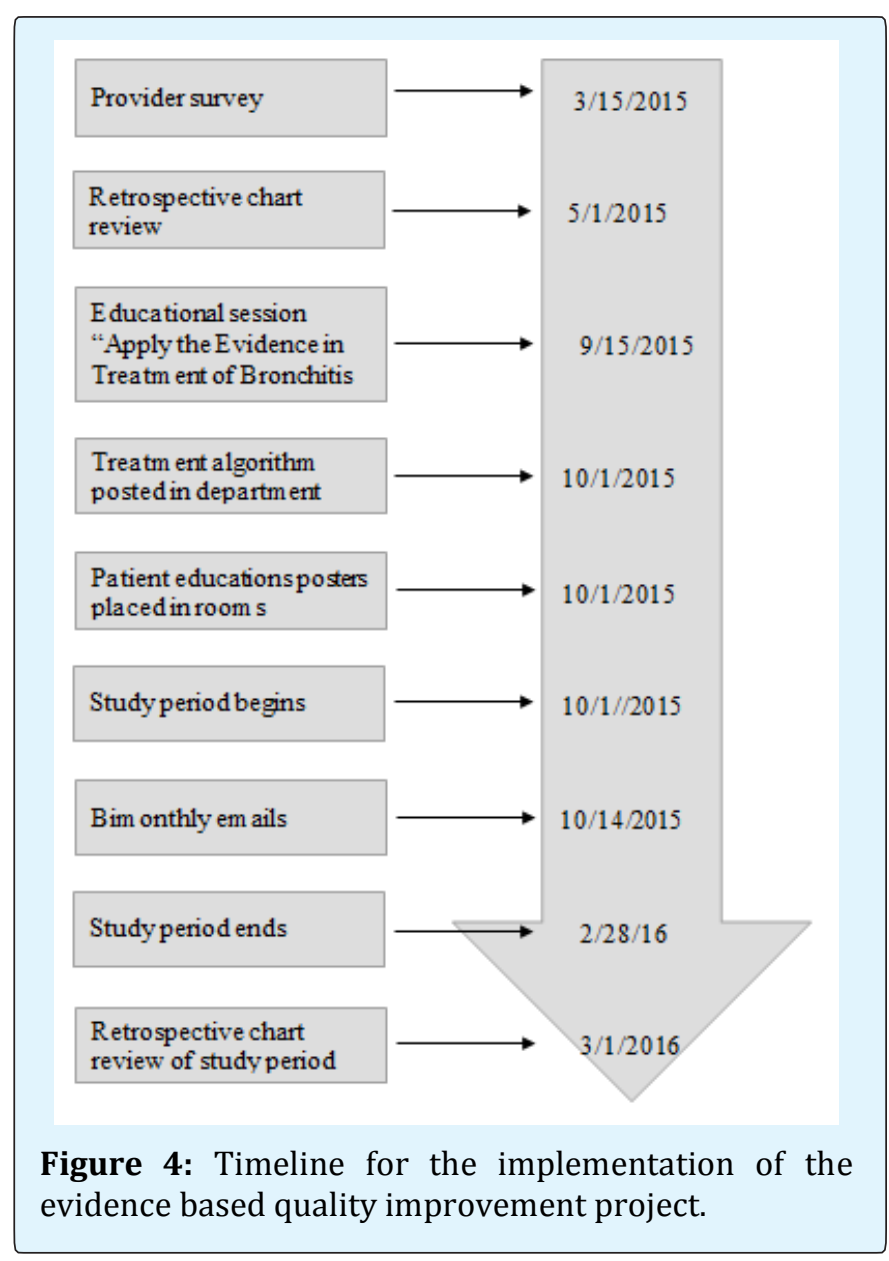

\section{Post Intervention}

The pre and post sample demographics did not show statistical significance between age, provider group and gender, race, or Emergency Severity Index (ESI) category (Table 1).

\begin{tabular}{|c|c|c|c|}
\hline & Pre-Intervention & Post Intervention & $\begin{array}{c}\text { Statistical significance } \boldsymbol{\alpha}<\mathbf{0 . 0 5} \\
\text { (Fischer's exact test) }\end{array}$ \\
\hline Patient meeting criteria & $\mathrm{n}=90$ & $\mathrm{n}=49$ & N.S \\
\hline Age & $37.83($ SD 10.85$)$ & $38.02($ SD 12.89$)$ & N.S \\
\hline \multicolumn{4}{|r}{} \\
\hline
\end{tabular}




\section{Nursing \& Healthcare International Journal}

\begin{tabular}{|c|c|c|c|}
\hline - MD & $26.7 \%(n=24)$ & $31 \%(\mathrm{n}=15)$ & N.S \\
\hline \multicolumn{4}{|c|}{ Gender of patient } \\
\hline - male & $30 \%(n=27)$ & $36.7 \%(\mathrm{n}=18)$ & N.S \\
\hline - female & $70 \%(n=63)$ & $63.3 \%(\mathrm{n}=31)$ & N.S \\
\hline Race & & & N.S \\
\hline - White & $43.3 \%(n=39)$ & $59.2 \%(n=29)$ & \\
\hline - Patient declined & $2.2 \%(n=2)$ & $4.1 \%(n=2)$ & \\
\hline $\begin{array}{c}\text { - Black or African } \\
\text { American }\end{array}$ & $51.1 \%(n=46)$ & $30.6 \%(n=15)$ & \\
\hline - Asian & $3.3 \%(n=3)$ & $2.0 \%(\mathrm{n}=1)$ & \\
\hline - American Indian & $0 \%(\mathrm{n}=0)$ & $4.1 \%(n=2)$ & \\
\hline \multicolumn{3}{|c|}{ ESI triage category } & N.S \\
\hline-2 & $3.3 \%(n=3)$ & $8.2 \%(n=4)$ & \\
\hline-3 & $42.2 \%(n=38)$ & $30.6 \%(n=15)$ & \\
\hline-4 & $54.4 \%(n=49)$ & $59.2 \%(n=29)$ & \\
\hline-5 & $0 \%(\mathrm{n}=0)$ & $2.0 \%(\mathrm{n}=1)$ & \\
\hline Antibiotics Yes & $50 \%(n=45)$ & $8.2 \%(n=4)$ & $p<0.0001$ \\
\hline Radiology Yes & $90 \%(n=81)$ & $67.3 \%(n=33)$ & $p=0.0012$ \\
\hline
\end{tabular}

Table 1: Sample characteristic pre and post intervention.

N.S. $=$ no significance found at $\alpha<0.05$.

A chart review done of the "intervention study period" found that $8.1 \%$ of patients received antibiotics for the diagnosis of acute uncomplicated bronchitis (Table 2). This reduction of $41.8 \% \quad[-0.418 \quad(-0.533,-0.271)]$ surpassed the goal of $20 \%$ and reached statistical significance with $\mathrm{p}<0.0001$. The relative risk of getting antibiotics post intervention was $0.163(0.062,0.427)$.

\begin{tabular}{|c|c|c|c|}
\hline \multicolumn{2}{|c|}{ Fischer's Exact test } & Two sample T Test of proportions (Yes) & Relative Risk (yes) \\
\hline \multicolumn{2}{|c|}{} & Difference (Lower 95 - Upper 95\%) & RR (Lower 95 - Upper 95\%) \\
\hline Antibiotics & $\mathrm{p}<0.0001$ & $-0.418(-0.533,-0.271)$ & $0.163(0.062,0.427)$ \\
\hline Radiology & $\mathrm{p}=0.0012$ & $-0.227(-0.369,-0.080)$ & $0.748(0.609,0.920)$ \\
\hline
\end{tabular}

Table 2: Statistical analysis.

During the same period, radiological studies ordered was reduced to $67.3 \%$. A reduction of $22.7 \%$ [- 0.227 ($0.369,-0.080)]$ which surpassed the goal of $20 \%$ and reached statistical significance with $\mathrm{p}<0.0012$. The relative risk of having a radiological study ordered post intervention was $0.748(0.609,0.920)$.

A post intervention survey of the providers was conducted. The survey was conducted using the Likert Scale, "1" being the most negative response and " 5 " being the most positive response. The return rate was $36.2 \%$. The provider mix was APPs 58.8\% and Physicians $41.2 \%$. The providers felt that the algorithm most met their learning style (4.65), followed by the live educational session (4.47). Regarding the promotion of evidencebased practice within the department, again they felt the algorithm met this need the most (4.65), followed by the live educational session (4.29). Most providers agree that their practice has changed as a result of this evidencebased project. (3.94). Please see Appendix 1 for the survey in its entirety.

\section{Discussion}

Through a multifaceted educational intervention, based on the best evidence for the diagnosis and treatment of acute uncomplicated bronchitis, it was determined that the intervention was successful in promotion of evidence-based care as measured by both the reduction of antibiotics prescribed and radiological studies ordered. Based on the evidence antibiotics are not indicated for the diagnosis of acute uncomplicated bronchitis. Furthermore, radiological studies are not needed to make this diagnosis if the patient has stable vital signs and no adventitious breath sounds. Reduction of antibiotics and radiological studies when not indicated 


\section{Nursing \& Healthcare International Journal}

should help reduce the problems of both antibiotic resistance and radiation exposure.

By using four different methods of education, at different times during the study period, the outcomes far surpassed those that were demonstrated in prior studies. In the review of the literature only one to two interventions were presented in each of the prior studies. Thus, suggesting that a multi-faceted educational approach has greater impact on providers' application of evidence-based practice in the management acute uncomplicated bronchitis.

Despite having a significant reduction in the number of radiological studies ordered, the amount of studies ordered remained quite high at $67 \%$. One possibility that is a likely contributor is due to having standing orders in the triage area. Nursing has the ability to order tests based on chief complaint. For future reference, one could consider changing the triage guidelines to avoid overordering of radiological studies. Further exploration of this possible contributor to high X-ray rates is needed.

\section{Limitations}

Findings are limited to an urban emergency department in the upper Midwest. Further limiting factors include: educational posters were only placed in three patient care rooms in the emergency department. Another limitation might have been the change from ICD 9 codes to QCD 10 codes. In addition, the post intervention group $(\mathrm{n}=49)$ was significantly smaller than the preintervention group $(n=90)$. In reviewing the surveillance data for this viral season from the Minnesota Department of Health's web site, it appears that it is a much later start to the influenza season (viral illness) than last year, which often corresponds with timing of increased rates of bronchitis. Minnesota Department of Health collaborates with health care providers and compares patients with "influenza like illness" to total number of patients seen in the clinic. In 2014-2015, patients presenting to clinics with influenza like illness peaked during week 52 with $8 \%$ of total patients. In 2015-2016, the number of patients presenting to clinics with influenza like illness peaked much later in the season (during week 10) and only at $4 \%$ [31,32]. This could account for the fewer numbers in the post intervention study. Another possibility for the reduced sample size in the post intervention group was possible changes in the diagnosis code. In a previous similar study by Ackerman, et al. [13] felt some clinicians were changing the diagnosis code to URI, so these patients would be excluded from the study.

Langer $\mathrm{K}$, et al. Applying the Evidence in Treatment of Acute Uncomplicated Bronchitis in Emergency Medicine. Nurs Health Care Int J 2019, 3(6): 000207.

\section{Conclusions}

Based on the study results a multi-faceted evidencebased practice intervention, in a large emergency care setting, was successful in promoting best practice in the diagnosis and management of acute uncomplicated bronchitis. A pre-intervention chart review lead to a successful Quality Improvement project during the given study period. Based on the results, providers focused on evidence-based practice thus allowing for appropriate management of acute uncomplicated bronchitis. Despite the study period coming to a close, providers can continue to practice based on the clinical care guidelines and literature provided. The educational posters remain in the examination rooms which can continue to be used for patient education purposes.

Given this intervention took place in a large emergency care setting; it would be worth disseminating the outcomes with other emergency departments in the area. Sharing this type of information will only help educate more providers and patients as acute bronchitis is a very common diagnosis seen in the emergency care setting. This type of education would benefit those practicing in a primary care environment as well. Based on the review of the clinical care guidelines, many were aimed towards the primary care environment. Research also shows that antibiotics are prescribed for upper respiratory infections, including acute bronchitis, within this clinical setting. This type of learning structure will reinforce evidence-based practice for all providers. Additionally, there is not a specific clinical care guideline aimed at care of the patient with acute uncomplicated bronchitis in the emergency care setting. Based on these results, a clinical care guideline could be developed and dispersed for use, based on the algorithm developed for this project. Given the extremely high success rate of this study's intervention at promoting best practice, additional educational efforts to promote evidence-based care in the management of other acute conditions maybe successful using this model, and should be explored.

\section{References}

1. Lee GC, Reveles KR, Attridge RT, Lawson KA, Mansi IA, et al. (2014) Outpatient antibiotic prescribing in the United States: 2000-2010. BMC Medicine 12: 96.

2. Tackett KL, Atkins A (2012) Evidence-based acute bronchtis therapy. J Pharm Pract 25(6): 586-590. 


\section{Nursing \& Healthcare International Journal}

3. Stone S, Gonzales R, Maselli J, Lowenstein SR (2000) Antibiotic prescribing for patients with colds, upper respiratory tract infections, and bronchitis: A national study of hospital-based emergency departments. Ann Emerg Med 36(4): 320-327.

4. Xu KT, Roberts D, Sulapas I, Martinez O, Berk J, et al. (2013) Over-prescribing of antibiotics and imaging in the management of uncomplicated URIs in emergency departments. BMC Emerg Med 13: 7.

5. Frieden $T$ (2013) Antibiotic resistance threats in the United States, 2013. Center for Disease Control and Prevention, pp: 5-113.

6. Jenkins S, Farrell D (2009) Increase in pneumococcus macrolide resistance, United States. Emerging Infectious Diseases 15(8): 1260-1264.

7. Get smart: Know when antibiotics work (2015) Center for Disease Control and Prevention.

8. Gonzalez ABD, Darby S (2004) Risk of cancer from diagnostic X-rays: Estimats for the UK and 14 other countries. Lancet 363(9406): 345-351.

9. Diederich S, Lenzen H (2000) Radioation exposure associated with imaging of the chest. Cancer 89(11): 2457-2460.

10. Mettler FA Jr, Bhargavan M, Faulkner K, Gilley DB, Gray JE, et al. (2009) Radiologic and Nuclear Medicine Studies in the United States and Worldwide: Frequency, Radiation Dose, and Comparison with Other Radiation Sources-1950-2007. Radiology 253(2): 520-531.

11. Hall EJ, Brenner DJ (2014) Cancer risks from diagnostic radiology. Br J Radiol 81(965): 362-378.

12. Brouwers MC, Kho ME, Browman GP, Burgers JS, Cluzeau F, et al. (2010) AGREE II: Advancing guideline development, reporting and evaluation in healthcare. CMAJ 182(18): E839-842.

13. Ackerman SL, Gonzales R, Stahl MS, Metlay JP (2013) One size does not filt all: Evaluating an intervention to reduce antibiotic presribing for acute bronchitis. BMC Health Serv Res 13(462): 1-9.

14. Smith SM, Fahey T, Smucny J, Becker LA (2014) Antibiotics for acute bronchitis (review). Cochrane Database Syst Rev (3): CD000245.
15. Rodnick JE, Gude JK (1988) The use of antibiotics in acute bronchitis and acute exacerbation of chronic bronchitis. West J Med 149(3): 347-351.

16. Gonzales R, Steiner JF, Lum A, Barrett PH Jr (1999) Decreasing antibioitc use in ambulatory practice: Impact of a multidimensional intervention of the treatment of uncomplicated acute bronchitis. JAMA 281(16): 1512-1519.

17. Altiner A, Berner R, Diener A, Feldmeier G, Köchling A, et al. (2012) Converting habits of antibiotic prescribing for respiratory tract infection in German primary care - the cluster randomized control CHANGE 2 trial. BMC Fam Pract 13: 124.

18. Cao AM, Choy JP, Mohanakrishnan LN, Bain RF, van Driel ML (2013) Chest radiographs for acute lower respiratory tract infections. Cochrane Database Syst Rev (12): CD009119.

19. Rogers EM (2003) Diffusion of Innovation. New York, NY: Free Press.

20. Llor C, Moragas A, Bayona C, Morros R, Pera H, et al. (2013) Effectiveness of anti-inflammatory treatment versus antibiotic therapy and placebo for patients with non-complicated bronchitis with purulent sputum. The BAAP Study protocol. BMC Pulm Med 11: 38.

21. Albert R (2010) Diagnosis and management of acute bronchitis. American Family Physician 82(11): 13451350.

22. Anderer $T$ (2011) An algorithm to improve appropriate antibiotic use for patients with acute bronchitis.

23. Mehrotra A, Wang MC, Lave JR, Adams JL, McGlynn EA (2008) Retail clinics, primary care physicians, and emergency department: A comparison of patient visits. Health Affairs 27(5): 1272-1282.

24. Michigan Quality Improvement Consortium. (2012). Managment of acute bronchitis in adults Michigan Quality Improvement Consortium.

25. Harris AM, Hicks LA, Qaseem A (2016) Appropriate antibiotic use for acute respiratory tract infection in adults: Advice for high-value care from the American College of Physicians and the Centers for Disease 
Control and Prevention. Ann Intern Med 164(6): 425434.

26. Aagaard EM, Gonzales R, Camargo CA Jr, Auten R, Levin SK, et al. (2010) Physician champions are key to improving antibiotic prescribing quality. Jt Comm J Qual Patient Saf 36(3): 109-116.

27. Harris RH, MacKenzie TD, Leeman Castillo B, Corbett KK, Batal HA, et al. (2003) Optimizing antibiotic prescribing for acute respiratory tract infections in an urban urgent care clinic. Journal of General Internal Medicine 18(5): 326-334.

28. (2014) Copyright and Permission Use of CDC Website Text and Materials.
29. Sall J (2016) JMP Statistical Software 12.

30. Polit D (2010) Statistics and Data Analysis for Nursing Research $2^{\text {nd }}($ edn), New Jersey: Pearson Education Inc.

31. (2016) Weekly Influenza and Respiratory Illness Activity Report.

32. Davidoff F, Batalden P, Stevens D, Ogrinc G, Mooney S (2008) Publication guidelines for quality improvement in health care: Evolution of the SQUIRE project. Quality Safe Health Care 17: i3-i9. 